\title{
Los primeros robots manipuladores aéreos
}

\author{
A.Ollero $^{1}$, A.Viguria ${ }^{2}$, I.Maza ${ }^{1}$, M.A. Trujillo ${ }^{2}$, G.Heredia $^{1}$, R.Cano ${ }^{2}$, F.Caballero ${ }^{1}$, F.Lasagni ${ }^{2}$, J.Á.Acosta $^{1}$, \\ S. Santos ${ }^{2}$ y J.A.Cobano ${ }^{1}$, \\ ${ }^{1}$ Grupo de Robótica, Visión y Control \\ Escuela Técnica Superior de Ingeniería (Universidad de Sevilla) \\ Camino de los Descubrimientos, $\mathrm{s} / \mathrm{n}$ \\ Isla de la Cartuja, 41092 Sevilla \\ \{aollero, imaza, guiller, fcaballero,jaar, jcobano\}@us.es \\ ${ }^{2}$ Centro Avanzado de Tecnologías Aeroespaciales \\ Parque Tecnológico y Aeronáutico de Andalucía. \\ C/ Wilbur y Orville Wright 17-19-21 \\ 41309 - La Rinconada (Sevilla) \\ \{aviguria,matrujillo,rcano,flasagni,ssantos\}@catec.aero
}

\section{Resumen}

En este articulo se describen los primeros robots manipuladores aéreos dotados con brazos robóticos de seis grados de libertad y capacidades de percepción y planificación que se han desarrollado en el mundo. Estos robots son el resultado del proyecto ARCAS (Aerial Robotics Cooperative Assembly System) coordinado por el primero de los autores y financiado por el Séptimo Programa Marco de la Unión Europea. Los resultados de este proyecto han comenzado a emplearse, entre otros, en el proyecto H2020 AEROARMS y en el español AEROMAIN. El artículo introduce las diferentes plataformas utilizadas en el proyecto y a continuación resume las principales características de los sistemas de control, percepción y planificación desarrollados. Se resume también la integración y validación del sistema

Palabras Clave: robots manipuladores aéreos, robots aéreos, sistemas aéreos no tripulados.

\section{INTRODUCCIÓN}

La robótica aérea está teniendo un importante auge en los últimos años, tanto desde el punto de vista de la investigación y desarrollo, como desde el punto de vista industrial. En la actualidad los principales congresos científicos de robótica, tales como los IEEE, ICRA y IROS, tienen múltiples sesiones de robótica aérea, y el número de artículos en las revistas especializadas ha crecido también de forma muy importante. El IEEE Technical Committee on Aerial Robotics and Unmanned Aerial Systems tiene varios cientos de miembros y ha sido nombrado en el pasado ICRA 2016 en Estocolmo como el Comité más activo. Por otra parte, solo en España, se cuenta actualmente con miles de empresas registradas como fabricantes de vehículos aéreos no tripulados y robots aéreos.
En la actualidad se conoce bien cómo controlar vehículos aéreos no tripulados tales como aviones de ala fija o multi-rotores. Se han desarrollado también numerosas técnicas de planificación de trayectorias y de percepción del entorno empleando como sensores cámaras convencionales, cámaras $3 \mathrm{D}$, láseres y otros sensores. La gran mayoría de estos robots aéreos permiten adquirir información, datos o imágenes, del entorno en múltiples aplicaciones, tales como la de vigilancia de recintos, la inspección y mantenimiento sin contacto, búsqueda y ayuda al rescate, exploración, etc. En [1] se muestra el estado del arte de los sistemas aéreos no tripulados y de sus aplicaciones. En un buen número de aplicaciones resulta conveniente el empleo de múltiples vehículos no tripulados homogéneos o heterogéneos [2].

Sin embargo, existen otras aplicaciones tales como la inspección y mantenimiento de instalaciones que requieren el contacto físico con objetos. En [3] se resume el estado de los sistemas aéreos no tripulados interaccionando entre sí y con en el entorno. Entre éstos, cabe mencionar los robots manipuladores aéreos cuyo desarrollo se propuso en 2011 con el proyecto ARCAS (Aerial Robotics Cooperative Assembly System). En este punto cabe distinguir entre los robots aéreos con dispositivos especiales para realizar una tarea específica, normalmente con muy pocas articulaciones (ver por ejemplo [4]-[6]), y los robots aéreos de propósito general para manipulación robótica aérea, tales como los que se presentan en este artículo desarrollados en el proyecto ARCAS.

En adelante, se presenta en primer lugar las características mecatrónicas, a continuación el control, seguido de secciones específicas dedicadas a la percepción y la planificación. Finalmente, se presentan las conclusiones y desarrollos futuros. 


\section{MECATRÓNICA}

Las plataformas aéreas desarrolladas en ARCAS son tanto helicópteros como multi-rotores. En la Figura 1 se muestra la evolución de las plataformas ARCAS empleadas en exteriores.

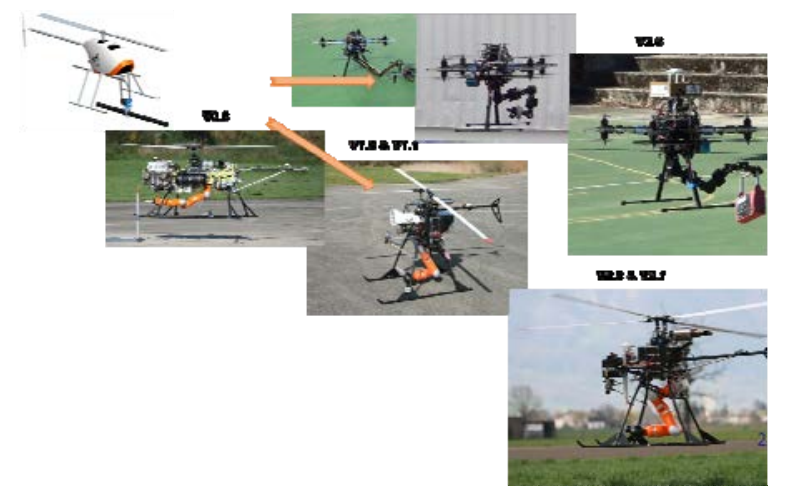

Figura 1: Robots aéreos para exteriores desarrollados en ARCAS.

Los helicópteros fueron desarrollados por el Centro Aeroespacial Alemán (DLR), mientras que los multirotores fueran desarrollados por el Grupo de Robótica, Visión y Control (GRVC) de la Universidad de Sevilla. No obstante, como se pondrá de manifiesto a continuación, en dichas plataformas se integraron métodos desarrollados por otros socios.

El primer prototipo era un helicóptero eléctrico dotado con una garra siguiendo conceptos similares a los empleados en [7]. A continuación, se desarrolló un helicóptero de mayores dimensiones capaz de integrar el conocido manipulador robótico LWR con siete grados de libertad (originalmente desarrollado por el DLR y posteriormente transferido a KUKA). Dicho robot manipulador tiene un peso total de aproximadamente $14 \mathrm{Kg}$ al que hay que añadir el de los objetos que puede transportar (hasta $7 \mathrm{Kg}$ ). El peso total resultante del helicóptero es de más de 100 Kg. Dicho helicóptero, con propulsión mediante motor de combustión, estaba en los límites de la carga de trabajo y presentaba problemas de seguridad. Posteriormente, se decidió cambiar la plataforma de helicóptero convencional por una de helicóptero Flettner con dos rotores principales en contra-rotación y sin rotor de cola. Esta plataforma es más eficiente que la del helicóptero convencional. Finalmente, se adoptó una plataforma Flettner con propulsión mediante turbina con un peso total algo mayor de $70 \mathrm{Kg}$. El incremento del peso relativo del brazo con respecto al peso total complicó el control, cuya problemática se aborda en la próxima sección.

Por lo que respecta a los multi-rotores, se han empleado plataformas con cuatro barras y dos rotores en contra-rotación en cada uno de ellas. Aunque la configuración es menos eficiente que la que emplea 8 rotores independientes, debido a las pérdidas producidas por los dos rotores montados en el extremo de la misma barra, suministra el empuje suficiente para cargar y operar el brazo robótico y es más compacta que la que emplea seis u ocho rotores en distintas barras. Se desarrolló un primer prototipo con un brazo robótico manipulador simple con tres grados de libertad que posteriormente se sustituyó por otro con un brazo robótico comercial con siete grados de libertad y un peso algo mayor de $2 \mathrm{~kg}$.

La elección de brazos robóticos con siete grados de libertad puede justificarse en términos de flexibilidad, acomodación y capacidad de reacción ante perturbaciones. De hecho, se ha podido demostrar experimentalmente que la manipulación mediante brazos con pocos grados de libertad tiende a generar pares desestabilizadores que pueden provocar fácilmente el vuelco de la plataforma aérea. Igualmente, se puso de manifiesto que, por ejemplo, con el helicóptero convencional el movimiento del brazo robótico fuera del plano vertical generaba pares cuya compensación con el sistema de control del helicóptero (rotor principal y cola) generaba oscilaciones [8]. El movimiento restringido al plano vertical hacía que el empleo de las siete articulaciones fuera especialmente beneficioso.

La Figura 2 muestra la evolución de las plataformas multi-rotores para interiores desarrolladas fundamentalmente en el Centro Avanzado de Tecnologías Aeroespaciales (CATEC).

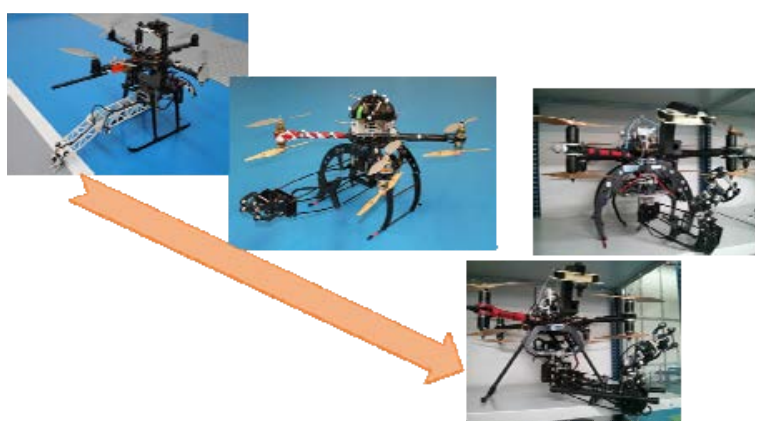

Figura 2: Plataformas ARCAS para interiores

La primera plataforma fue un multi-rotor comercial (Pelican, AsTech) al que se dotó de un brazo muy ligero, con solo dos grados de libertad, de material polimérico, construido mediante Fabricación Aditiva o Additive Manufacturing (AM). A continuación se desarrolló una plataforma de características similares, aunque algo más pequeña, que la de la Figura 1, con cuatro barras con dos rotores cada una. Se la dotó con un brazo robótico muy ligero, con dos grados de libertad, con varillas pretensadas y rigidizadores de material compuesto. Posteriormente se lo sustituyó por un brazo de seis grados de libertad construido también con varillas pretensadas y rigidizadores de 
material compuesto. En la Figura 3 se muestra la configuración del manipulador y su base.

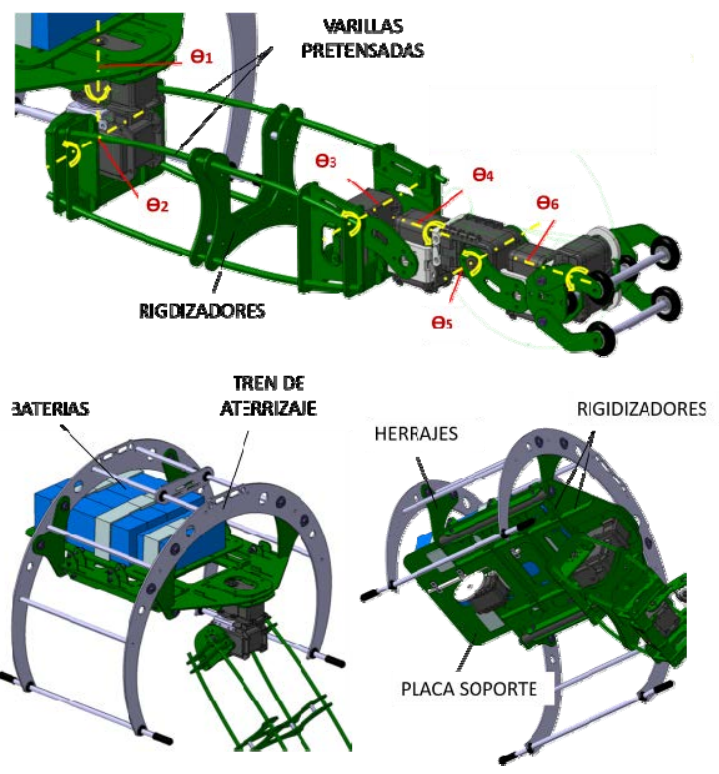

Figura 3: Brazo manipulador con 6 grados de libertad con un efector final diseñado para el agarre, transporte e inserción de barras. En la parte inferior se muestra el soporte del brazo.

Un componente relevante de esta plataforma es el que permite desplazar las baterías en dirección contraria al movimiento del brazo ayudando a compensar el desplazamiento del centro de gravedad (Patente P201330537).

Finalmente se desarrolló una nueva plataforma en la que se integró en el brazo un sensor de fuerza/par.

\section{SISTEMAS DE CONTROL}

El control de un robot manipulador aéreo es un problema complejo si las dimensiones y peso del manipulador son relevantes con respecto al peso de la plataforma, tal como sucede en los manipuladores aéreos mencionados en el apartado anterior. En efecto, en estas plataformas el movimiento del brazo y la interacción con objetos en el entorno genera pares que tienden a desestabilizar la plataforma aérea que además está sometida a perturbaciones debidas a efectos aerodinámicos generados por las superficies próximas.

En general, respecto a la arquitectura de control, cabe distinguir entre los siguientes enfoques:

1. La plataforma aérea y el brazo se consideran como dos entidades diferentes y se trata de disminuir los efectos generados por el movimiento de una sobre la otra.

2. Se consideran dos entidades diferentes pero en el control de cada una de ellas se tiene en cuenta la información del movimiento de la otra.

3. La plataforma aérea y el manipulador se consideran como una entidad única con un modelo dinámico completo con múltiples grados de libertad que se pretenden controlar de forma conjunta.

En el primero de los casos pueden aplicarse técnicas de control de impedancia. Se definen bucles de control de impedancia, tanto para los movimientos rotacionales como para los lineales. Las referencias para el efector final se determinan basándose en las fuerzas generalizadas generadas en la muñeca del manipulador. Si se dispone de un sensor de fuerzas y pares en la muñeca, es posible cerrar directamente el bucle de control. En caso contrario, es posible estimar las fuerzas y pares que actúan sobre el vehículo [9].

En [10] se introduce un controlador que considera el acoplamiento cinemático empleando el control de orientación del helicóptero como un grado de libertad adicional del manipulador. Asimismo, se diseña un sistema de control midiendo las fuerzas y pares en la base del manipulador para compensar el movimiento de éste teniendo en cuenta el acoplamiento dinámico. Siguiendo la estrategia 2, el controlador del multirotor considera el desplazamiento del centro de gravedad y la variación de los momentos de inercia debido al movimiento del manipulador. Por otra parte, el controlador del brazo considera el estado de la plataforma aérea para estabilizar el efector final del manipulador. En [11] se presenta esta estrategia que se ilustra en la Figura 4.

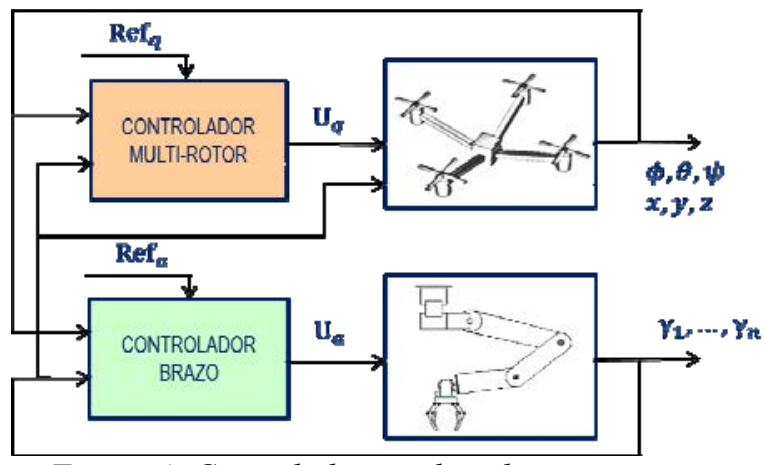

Figura 4: Controlador empleando interacciones

Se emplea un término de prealimentación para compensar los pares generados por el manipulador y el objeto manipulado. Asimismo, en el controlador de la plataforma aérea, se utiliza un controlador basado en "Backstepping", con parámetros variables para tener en cuenta el movimiento del manipulador, y un término integral. En [12] se presenta un controlador similar para la plataforma aérea y un controlador basado en admitancia para el brazo. Este controlador genera las posiciones deseadas para el efector final del manipulador. En la Figura 5 se muestran los resultados de experimentos de posicionamiento y agarre de objetos. Las mismas técnicas se emplean en [13] para control de un helicóptero con el brazo manipulador robótico. 


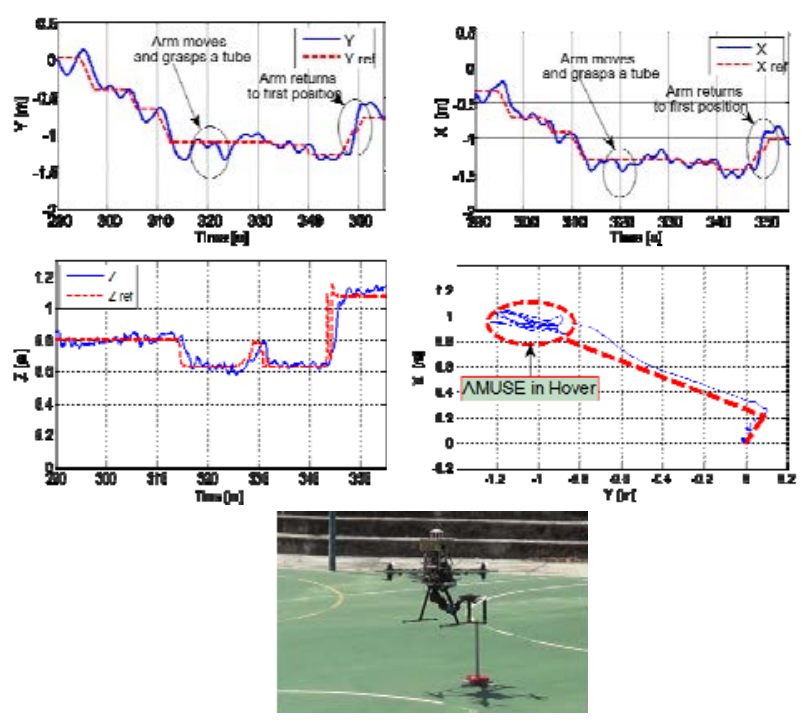

Figura 5: Posicionamiento y agarre de objetos de multi-rotor con brazo de 7 grados de libertad

Finalmente, siguiendo el enfoque 3 pueden emplearse también estrategias de control basadas en "Backstepping" con parámetros variables o, en general, sistemas de control basados en pasividad (PBC). Se ha empleado un controlador pasivo con la técnica IDA-PBC (Interconnection and Damping Assignment Passivity-Based Control) [14] garantizando la estabilidad y robustez para movimientos en 2D. En la Figura 6 se ilustra el sistema de control completo implementado de forma coordinada con el control del brazo [15].

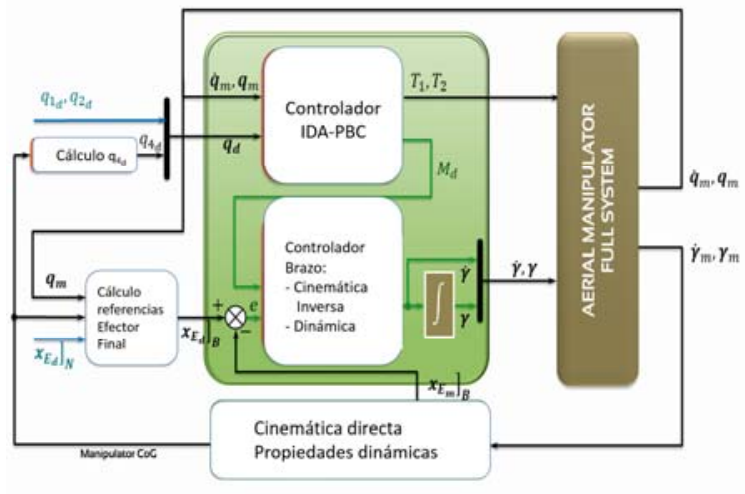

Figura 6: Control basado en IDA-PBC

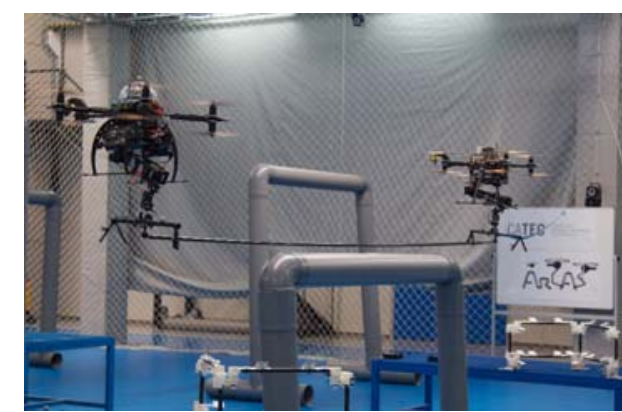

Figura 7: Transporte cooperativo mediante dos manipuladores
Asimismo, en ARCAS, se ha trabajado en el control cooperativo de dos manipuladores transportando un objeto como se muestra en la Figura 7.

En la Figura 8 se muestra el esquema de control cooperativo mediante impedancia. El bucle externo modifica la trayectoria deseada del objeto de acuerdo con un comportamiento masa-amortiguador-muelle (impedancia mecánica) generando una trayectoria deseada para el objeto. En [16] se describe el método desarrollado.

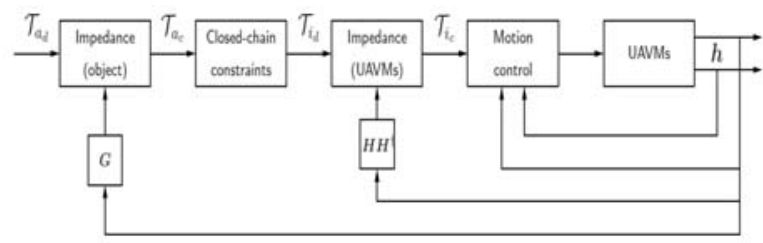

Figura 8: Control cooperativo mediante impedancia

\section{PERCEPCIÓN}

El sistema de percepción de ARCAS incluye funcionalidades de distintos niveles. La Universidad Politécnica de Cataluña (UPC) ha liderado el desarrollo de estas funcionalidades con la colaboración de la Universidad de Sevilla, la Universidad de Nápoles y el DLR.

La UPC ha desarrollado sistemas de estimación de la posición y orientación empleando imágenes obtenidas en robots aéreos incluso cuando no existe calibración previa [17]. Para ello se emplean clasificadores entrenados con imágenes difuminadas, de baja resolución e incluso con oclusiones. Se han desarrollado también métodos de reconocimiento de objetos empleando Random Ferns [18] y métodos invariantes ante rotaciones $3 \mathrm{D}$. Asimismo, se han desarrollado métodos de análisis y clasificación de terrenos [19] con objeto de seleccionar automáticamente la ubicación de la estructura

El DLR ha desarrollado técnicas para la generación de mapas 3D con el método "Semi Global Matching" empleando pares estéreos y FPGA para el procesamiento de imágenes.

Por otra parte, se han desarrollado también métodos para el seguimiento fiable de objetos en 3D involucrando técnicas de estimación y seguimiento, y métodos de realimentación visual cerrando los bucles de control para el agarre de objetos y el ensamblado [20]. Estos métodos se han implementado también empleando imágenes no calibradas [21]. La implementación experimental se ha realizado empleando marcadores visuales para detectar e identificar objetos. Estas técnicas han permitido implementar métodos de realimentación visual. En la Figura 9 se muestran fotos de experimentos.

Por otra parte, en la Universidad de Sevilla, se han desarrollado métodos de estimación simultánea de posición y construcción de mapas empleando sensores que miden solo distancia (RO-SLAM) [22] 
con tecnologías de intensidad de la señal de radio y de tiempo de vuelo. Para ello se integran en las barras emisores de radio que permiten su localización con el robot aéreo. En la Figura 10 se muestran resultados del método. Es posible también realizar la fusión de las estimaciones RO-SLAM y visuales [23].

Asimismo, se han desarrollado métodos de percepción cooperativa combinando información de múltiples robots aéreos incluyendo fusión sensorial. En este caso se integran percepciones obtenidas con distintos robots aéreos evitando los problemas de oclusiones que se tienen empleando un único robot.
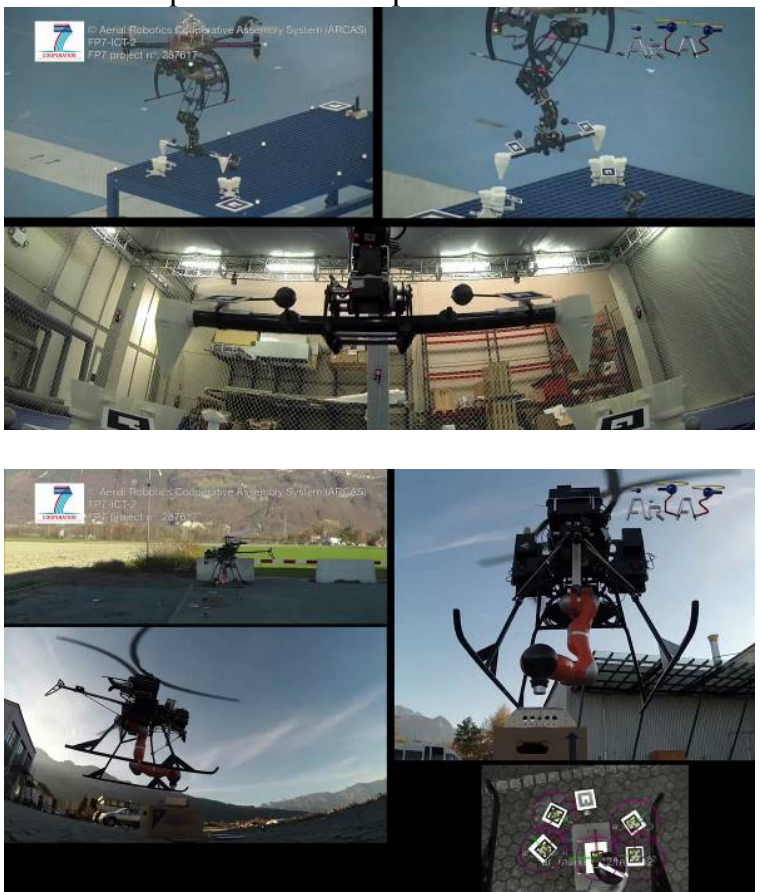

Figura 9: Experimento de realimentación visual en interiores (ver parte superior) y exteriores (ver parte inferior).

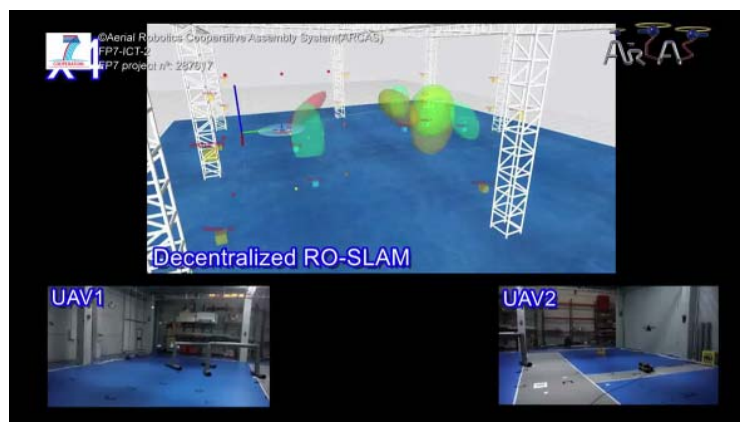

Figura 10: Aplicación de métodos RO-SLAM descentralizado. Las elipses en verde indican la convergencia en la estimación de la posición de las barras.

\section{PLANIFICACIÓN}

El sistema de manipulación robótica de ARCAS incluye funcionalidades de planificación autónoma para múltiples robots cooperativos. Se incluyen tres fases:
- planificación de la misión, que en el caso de ARCAS es el ensamblado de una estructura mediante barras

- planificación de tareas en un contexto multirobot

- planificación de las trayectorias de los robots aéreos

La arquitectura global del sistema de planificación se muestra en la Figura 11.

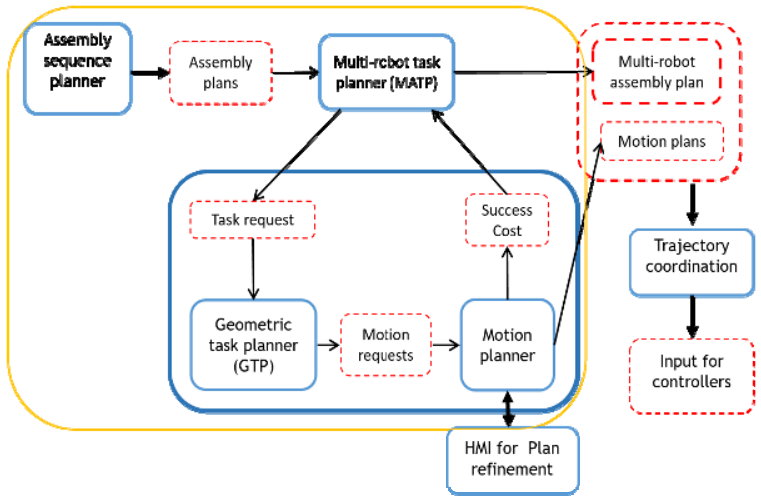

Figura 11: Arquitectura global del sistema de planificación.

El planificador de ensamblaje desarrollado por la Universidad de Sevilla está basado en la librería Bullet, que permite considerar la gravedad, las fricciones, el balance de masas, etc en cada fase del proceso de ensamblaje. Se realiza un modelado mediante grafos no direccionales que permiten obtener planes empleando la técnica de "ensamblado a partir del desensamblado" seleccionando la secuencia que optimiza un índice. En la Figura 12 se muestra el diagrama de bloques que permite determinar una secuencia de ensamblaje a partir de un modelo $\mathrm{CAD} 3 \mathrm{D}$ de la estructura que se pretende ensamblar.

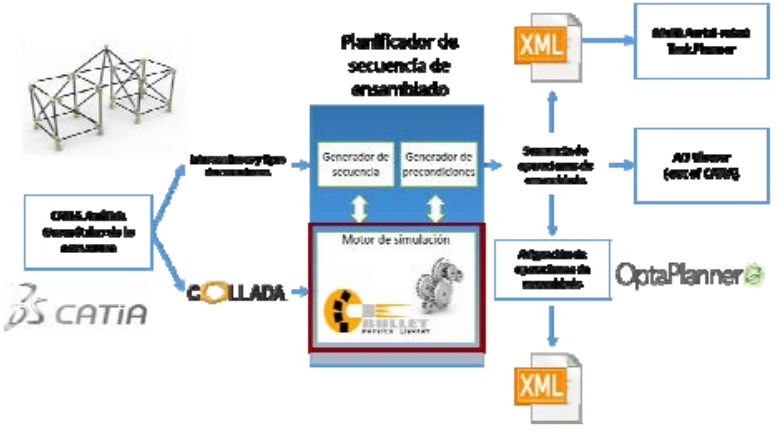

Figura 12: Planificación del ensamblado de una estructura en ARCAS.

Los resultados de la planificación se representan mediante una gramática de ensamblado para su tratamiento mediante el planificador de tareas que ha sido desarrollado por el equipo del CNRS.

Este equipo aplica métodos que integran tanto la planificación simbólica como la geométrica permitiendo producir tareas para varios robots 
trabajando en paralelo. El resultado de este planificador es una secuencia de puntos de paso para cada robot manipulador aéreo. Dicha secuencia es la entrada para el planificador de movimientos, también desarrollado por el CNRS. Este planificador calcula caminos de buena calidad para el robot manipulador aéreo empleando una técnica multi-árbol basada en el conocido algoritmo de planificación RRT (Rapidly Exploring Random Tree) [25][26]. El nuevo método permite considerar restricciones cinemáticas $y$ dinámicas en el movimiento [27] e incorpora un nuevo mecanismo de muestreo que permite reducir el tiempo de cálculo mediante grafos bi-direccionales RRT y métodos PRM bidireccionales.

En la última fase del proyecto ARCAS se consiguió integrar el planificador de ensamblado, el de tareas y el de movimientos (recuadro amarillo en Figura 11) de forma que solo se asignan acciones a robots que pueden ejecutarlas teniendo en cuenta las posibles colisiones con el entorno y con otros robots mediante trayectorias que tienen en cuenta las características del robot. De esta forma, el planificador de ensamblado solo propone los planes que tienen más posibilidades de ejecutarse de forma apropiada.

Como se ilustra en la Figura 11 existe la posibilidad de que el operador intervenga para refinar los planes empleando para ello un método desarrollado por la Universidad Politécnica de Cataluña.

Los caminos obtenidos mediante el planificador de movimiento se analizan para verificar que no se producen colisiones entre robots. Para ello el equipo de la Universidad de Sevilla ha desarrollado métodos de evitación de colisiones que permiten modificar de forma óptima las velocidades de los distintos robots minimizando un índice de coste de separación de los planes [28]. De esta forma, se tienen en cuenta las trayectorias espaciales computadas anteriormente.

Asimismo también se han aplicado métodos reactivos basados en ORCA (Optimal Reciprocal Collision Avoidance), que trabajan en el espacio de velocidades, teniendo en cuenta restricciones cinemáticas, compartiendo el esfuerzo de evitar una colisión entre los robots involucrados en una posible colisión, y minimizando las diferencias con respecto a las velocidades de crucero planificadas [29]. La implementación del nuevo método incluye como parte novedosa el considerar obstáculos estáticos 3D de la estructura del entorno. Este método se probó en simulación con hasta 20 robots aéreos en diferentes escenarios y hasta 8 en el escenario que se indica en el aparado siguiente implementándose en la demostración integrada final que se describe a continuación con tres cuatro multi-rotores.

\section{INTEGRACIÓN Y VALIDACIÓN}

Los métodos desarrollados en los apartados anteriores se han integrado $\mathrm{y}$ validado en tres escenarios.
El primero es el testbed de interiores del CATEC con dimensiones $16 \times 15 \times 5$ metros dotado del sistema VICON. En este escenario, se han aplicado los robots manipuladores aéreos desarrollados en el CATEC (ver Figura 2), integrándose todos los métodos anteriores, realizando una demostración consistente en el montaje de dos subestructuras con barras cortas, transportables y ensamblables mediante un único robot manipulador aéreo, que finalmente se conectaron mediante una barra que se transporta y despliega mediante dos robots como se muestra en la Figuras 7 y 13. Para ello se han desarrollado barras y conectores apropiados empleando técnicas de ALM.
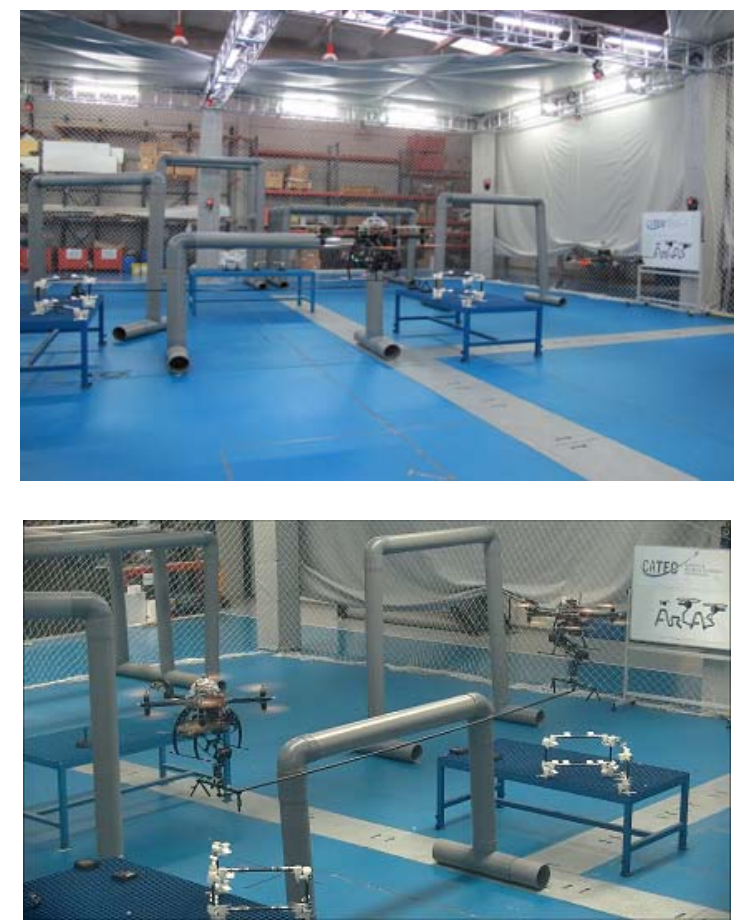

Figura 13: Demostración integrada indoor de ARCAS

Los pasos de esta demostración son:

1. Aplicación del método de Range-Only SLAM para mapeado y localización de las barras con emisores de radio

2. Planificación y cálculo de trayectorias de robot manipulador aéreo

3. Cálculo de trayectorias de robots auxiliares con cámaras

4. Ejecución de trayectorias de robots aéreos con detección y evitación de colisiones mediante ORCA. Agarre y transporte de barra corta

5. Ensamblado de barra y vuelta al paso 2 hasta que todas las barras cortas se han ensamblado

6. Agarre, transporte y despliegue de barra larga conectando las dos estructuras incluyendo detección y evitación de colisiones durante el transporte. 
En el vídeo del año 4 de ARCAS (https:/www.youtube.com/watch? $\mathrm{v}=\mathrm{Cs}$ lR9ArrgBA) se incluye esta demostración.

El segundo escenario es el de exteriores. En este se han aplicado tanto helicópteros (ver Figura 1 y 9 inferior) como multi-rotores de mayores dimensiones (ver Figura 5), realizándose experimentos de percepción del entorno, posicionamiento preciso $\mathrm{y}$ manipulación empleando realimentación visual.

Finalmente cabe mencionar que los métodos desarrollados en ARCAS se han aplicado también en un escenario de robótica orbital en el que se han simulado dos satélites, uno de ellos dotado con el manipulador LWR realizando operaciones de servicio al otro satélite. Los movimientos de los satélites se simulan mediante dos robots manipuladores de mayores dimensiones cuyos efectores finales mantienen a los mock-ups de los satélites.

\section{CONCLUSIONES Y DESARROLLOS FUTUROS}

El proyecto ARCAS ha desarrollado, por primera vez a nivel mundial, robots manipuladores aéreos, basados tanto en helicópteros como en multi-rotores, con brazos robóticos de 6 y 7 grados de libertad abriendo el camino de otros robots basados en multirotores que se han desarrollado posteriormente en EEUU, Japón y Corea. Existen también otros intentos que han fracasado por minusvalorar las dificultades del desarrollo de los robots manipuladores aéreos y su control.

ARCAS es el único sistema en el mundo de manipulación robótica aérea que integra capacidades autónomas avanzadas de percepción y planificación.

En la Web de ARCAS http://www.arcas-project.eu/ se incluyen otros videos y las 144 publicaciones científicas que ha generado el proyecto.

Puede considerarse que ARCAS, que ha sido evaluado como "Excelente" por la Comisión Europea, ha creado la Robótica Aérea de Manipulación entendida como área científica en la que se abordan problemas generales de manipulación empleando robots aéreos.

La tecnología desarrollada en ARCAS continúa mejorándose en múltiples proyectos, entre los que cabe mencionar, el proyecto Europeo de H2020 AEROARMS (http://www.aeroarms-project.eu/) en el que se están desarrollando robots aéreos con múltiples brazos y capacidades avanzadas de manipulación, y que incluye la aplicación a la inspección y mantenimiento industrial, contemplando específicamente aplicaciones en industrias petroquímicas. Asimismo, en el proyecto español AEROMAIN, se contempla la aplicación de la manipulación robótica aérea para la inspección y mantenimiento de aerogeneradores eólicos.
Finalmente, en el proyecto H2020 AEROBI, se aplicarán los resultados de ARCAS para la inspección de puentes.

\section{Este artículo se presenta con el vídeo:}

https://www.youtube.com/watch?v=QmNsnjKA7w8 en el cual se resumen los resultados del proyecto. El vídeo podría presentarse en la sesión general prevista en las Jornadas de Automática.

\section{Agradecimientos}

Este trabajo ha sido financiado parcialmente por el Proyecto Europeo del FP7 ARCAS (ICT-2011287617) y por el H2020 AEROARMS (ICT-232014-644271), así como por el proyecto nacional AEROMAIN DPI2014-59383-C2-1-R del Ministerio de Economía y Competitividad.

Los autores agradecen la colaboración de todos los socios de ARCAS, que además de CATEC y la Universidad de Sevilla son: Centro Aeroespacial Alemán (DLR), Universidad de Nápoles y asociados (Universidad de Cassino y Universidad de Basilicata), CNRS (Laboratorio LAAS), Universidad Politécnica de Cataluña (IRI-CSIC como asociado), Alstom Inspection Robotics y Space Tech.

\section{Referencias}

[1] K. Valavanis y G. Vatsevanos. Handbook of Unmanned Aerial Systems. Springer. 2014.

[2] A. Ollero and I. Maza. Multiple Heterogeneous Aerial Vehicles" Springer tracts in Advanced Robotics. Springer, Berlín Heidelberg 2007.

[3] K. Kondak, A. Ollero, I. Maza, K. Krieger, A. Albu-Schaeffer, M. Schwarzbach and M. Laiacker. "Unmanned Aerial Systems Physically Interacting with the Environment. Load Transportation, Deployment and Aerial Manipulation" Handbook of Unmanned Aerial Systems. Springer. 2014.

[4] Lindsey, Q., Mellinger, D., and Kumar, V. "Construction of cubic structures with quadrotor teams". In Proceedings of Robotics: Science and Systems, Los Angeles, USA. 2011

[5] Mellinger, D., Lindsey, Q., Shomin, M., and Kumar, V. Design, modeling, estimation and control for aerial grasping and manipulation. In IROS, pages 2668-2673. IEEE. 2011.

[6] Michael, N., Fink, J., and Kumar, V. Cooperative manipulation and transportation with aerial robots. Autonomous Robots, 30(1):73-86. 2011.

[7] Pounds, P. E. I., Bersak, D. R., and Dollar, A. M. Grasping from the air: Hovering capture and load stability. Proc.ICRA, pp2491-2498, 2011.

[8] K. Kondak, K. Krieger, A. Albu-Schaeffer, M. Schwarzbach, M. Laiacker, I. Maza, A. Rodriguez-Castano and A. Ollero, "Closed loop 
behavior of an autonomous helicopter equipped with a robotic arm for aerial manipulation tasks", International Journal of Advanced Robotic Systems.

[9] F. Ruggiero, J. Cacace, H. Sadeghian, V. Lippiello, "Impedance control of VToL UAVs with a momentum-based external generalized forces estimator", 2014 IEEE Int. Conf. on Robotics and Automation, June 2014.

[10] K. Kondak, F. Huber, M. Schwarzbach, M. Laiacker, D. Sommer, M. Bejar, A. Ollero, "Aerial manipulation robot composed of an autonomous helicopter and a 7 degrees of freedom industrial manipulator", Proceedings of the 2014 IEEE International Conference on Robotics and Automation, June 2014.

[11] A.E. Jimenez-Cano, J. Martin, G. Heredia, R. Cano, A. Ollero, "Control of an aerial robot with multi-link arm for assembly tasks", Int. Conf. on Robotics and Automation (ICRA 2013), May 6 - 10, 2013.

[12] G. Heredia, A.E. Jimenez-Cano, I. Sanchez, D. Llorente, V. Vega, J. Braga, J.A. Acosta and A. Ollero, "Control of a Multirotor Outdoor Aerial Manipulator", Proc. IROS 2014, Sept. 2014.

[13] A.E. Jimenez-Cano, G. Heredia, M. Bejar, K. Kondak and A. Ollero, "Modelling and control of an aerial manipulator consisting of an autonomous helicopter equipped with a multilink robotic arm", Journal of Aerospace Engineering, 7th December, 2015.

[14] J.Á. Acosta, M.I. Sánchez and A. Ollero, Robust Control of Underactuated Aerial Manipulators via IDA-PBC, 53rd IEEE Conference on Decision and Control (CDC 2014), 15th December, 2014.

[15] M.I. Sánchez, J.Á Acosta and A. Ollero, "Integral action in first-order Closed-Loop Inverse Kinematics. Application to aerial manipulators," 2015 IEEE International Conference on Robotics and Automation (ICRA), Seattle, WA, 2015, pp. 5297-5302.

[16] G. Muscio, F. Pierri, M.A.Trujillo E. Cataldi, G. Giglio, G. Antonelli, F.Caccavale, A. Viguria, S.Chiaverini, A. Ollero. "Experiments on Coordinated Motion of Aerial Robotic Manipulators". Proc ICRA 2016. Stockholm.

[17] A. Penate-Sanchez, J. Andrade-Cetto and F. Moreno-Noguer. "Exhaustive linearization for robust camera pose and focal length estimation". IEEE Transactions on Pattern Analysis and Machine Intelligence, 35(10): 2387-2400, 2013.

[18] M. Villamizar, J. Andrade-Cetto, A. Sanfeliu and F. Moreno-Noguer. "Bootstrapping boosted random Ferns for discriminative and efficient object classification". Pattern Recognition, 45(9): 3141-3153, 2012.
[19] A.Santamaria-Navarro, E.H.Teniente, M.Morta, J.Andrade-Cetto, "Terrain classification in complex three-dimensional outdoor environments", Journal of Field Robotics, June 2014.

[20] R. Mebarki, V. Lippiello, B. Siciliano, Nonlinear visual control of unmanned aerial vehicles in GPS-denied environments, IEEE Transactions on Robotics, 31 (4), 1004-1017, 2015, 2015.

[21] À. Santamaria-Navarro, J. Andrade-Cetto, "Uncalibrated image-based visual servoing", 2013 IEEE International Conference on Robotics and Automation.

[22] F.R. Fabresse, F. Caballero and A. Ollero. "Decentralized Simultaneous Localization and Mapping for Multiple Aerial Vehicles Using Range-Only Sensors. International Conference on Robotics and Automation (ICRA 2015). Seattle, USA, 26-30 May 2015.

[23] F.R. Fabresse, F. Caballero, I. Maza, A. Ollero, "Localization and mapping for aerial manipulation based on range-only measurements and visual markers", 2014 IEEE Int. Conf. on Robotics and Automation, 2014.

[24] J. Munoz-Morera, I. Maza, C. J. FernandezAguera, F. Caballero and A. Ollero, Assembly planning for the construction of structures with multiple UAS equipped with robotic arms, Proceedings of the 2015 International Conference on Unmanned Aircraft Systems (ICUAS 2015), 9th June, 2015.

[25] D. Devaurs, T. Siméon, J. Cortés, "Parallelizing RRT on large-scale distributed-memory architectures", IEEE Transactions on Robotics, Apr. 2013.

[26] D. Devaurs, T. Siméon, J. Cortés, Optimal path planning in complex cost spaces with samplingbased algorithms, IEEE Transactions on Automation Science and Engineering, 2014.

[27] A. Boeuf, J. Cortés, R. Alami and T. Siméon, Enhancing sampling-based kinodynamic motion planning for quadrotors, 2015 IEEE/RSJ International Conference on Intelligent Robots and Systems, Sept 28 - Oct 03, 2015.

[28] D. Alejo, J. A. Cobano, G. Heredia, A. Ollero, "Collision-free 4D trajectory planning in unmanned aerial vehicles for assembly and structure construction", Journal of Intelligent and Robotic Systems, Vol. 73, pp. 783-795, 2014.

[29] D. Alejo, J. Cobano, G. Heredia, and A. Ollero, "A reactive method for collision avoidance in industrial environments," Journal of Intelligent and Robotic Systems, pp. 1-14, 2016. 